\title{
Evaluación de usabilidad de un sistema de administración de cursos basado en la plataforma Lingweb
}

\author{
Usability evaluation of a course management system based on Lingweb platform
}

\author{
Javier M. Reyes Vera ${ }^{1 *} \quad$ Martha Isabel Berdugo Torres ${ }^{2} \quad$ Liliana Machuca Villegas $^{3}$
}

Recibido 26 de mayo de 2015, aceptado 27 de noviembre de 2015

Received: May 26, 2015 Accepted: November 27, 2015

\begin{abstract}
RESUMEN
El artículo presenta unos criterios metodológicos para evaluar la usabilidad en los Sistemas de Administración de Sursos (CMS). Están basados en métodos tradicionales de evaluación de usabilidad. A partir de estos métodos se originaron nuevos que fueron mezclados con los tradicionales para evaluar no solo los elementos que componen la usabilidad sino la funcionalidad y el aspecto pedagógico de los CMS. Estos criterios se aplicaron en la evaluación de la usabilidad de la plataforma de aprendizaje Lingweb que se realizó mezclando métodos y aprovechando al máximo las sesiones con Tester Users (TU), lo que generaron insumos importantes para ser abordados en un análisis riguroso y detallado de las diferentes características encontradas en la plataforma Lingweb desde la perspectiva de la usabilidad, de la funcionalidad y de la pedagogía. Los resultados obtenidos en esta evaluación desde el análisis de la eficiencia de la plataforma son descritos en el artículo.
\end{abstract}

Palabras clave: Evaluación de usabilidad, evaluación de eficiencia, heurísticas, Sistema de Administración de Cursos (CMS).

\section{ABSTRACT}

This paper presents methodological criteria for evaluating usability of course management systems $(C M S)$. These criteria are based on traditional methods of usability evaluation within a mixed approach; some new methods emerged while evaluating the usability as well as the pedagogical and functional features of a CMS called Lingweb. In this case study, Tester User (TU) sessions were carried out and fully exploited. They brought us important elements which led us to make a rigorous and detailed analysis from a three-fold usability-functionality-pedagogy perspective. We describe and discuss here the results of the analysis of Lingweb efficiency.

Keywords: Usability evaluation, efficiency evaluation, heuristics, courses management systems.

\section{INTRODUCCIÓN}

El proceso de desarrollo de software implica una serie de pasos que son necesarios para el desarrollo de cualquier aplicación informática [1]. En años recientes, el usuario final ha sido considerado como parte importante del proceso debido a que es la persona que interactúa con los elementos funcionales de una aplicación [2]. La calidad del producto software se define como el grado en el

1 Departamento de Diseño. Facultad de Artes Integradas. Universidad del Valle. Calle 13, \# 100-00, of 4018, edif 382. Cali, Colombia. E-mail: javier.reyes@correounivalle.edu.co

2 Escuela de Ciencias del Lenguaje. Facultad de Humanidades. Universidad del Valle. Calle 13, \# 100-00, of 4018, edif 382. Cali, Colombia. E-mail: martha.berdugo@ correounivalle.edu.co

3 Escuela de Ingeniería de Sistemas y Computación. Facultad de Ingeniería. Universidad del Valle. Calle 13, \# 100-00, of 4018, edif 382. Cali, Colombia. E-mail: Liliana.machuca@ correounivalle.edu.co

* Autor de correspondencia 
cual un producto satisface las necesidades implícitas y explícitas cuando es utilizado bajo condiciones específicas [3]; uno de sus elementos es desde luego la usabilidad [3-4].

La usabilidad se define como "la eficacia, eficiencia y satisfacción con la que un producto permite alcanzar objetivos específicos a usuarios específicos en un contexto de uso específico" [5]. Para evaluar la usabilidad de una aplicación informática es necesario emplear un conjunto de herramientas que permitan la medición de estos elementos de manera apropiada desde el punto de vista cualitativa y cuantitativa. Existe un amplio abanico de métodos de evaluación de usabilidad (e. g., métodos de inspección, indagación y test) que pueden ser usados dependiendo del contexto y las características de un proyecto. Generalmente estos métodos se combinan para obtener mejores resultados [6], [4, 7-10]. Así, dentro de los métodos de inspección se pueden combinar la evaluación heurística $[7,11,2]$ y la caminata o recorrido cognitivo [12].

Como ejemplo de aplicación de la usabilidad dentro del desarrollo de software (inspección), en [13] han propuesto la incorporación de la usabilidad dentro del ciclo de vida del desarrollo del software, cuyo objetivo consiste en que los desarrolladores apliquen un conjunto de artefactos para realizar una evaluación de usabilidad gradual en diversos módulos y hacer las modificaciones necesarias antes de que llegue al usuario final.

Otra preocupación recurrente en los investigadores, además de que la evaluación de usabilidad es un campo relativamente nuevo, es el alto costo de la infraestructura tecnológica para realizarla. Los grupos CAMALEÓN (Grupo de investigación en usabilidad y adaptatividad) y EILA (Equipo de Investigación en Lingüística Aplicada) han desarrollado y adaptado un conjunto de métodos en el marco de varios proyectos de investigación, cuya finalidad es estar en consecuencia con las dinámicas expuestas en los párrafos anteriores pero teniendo en cuenta que debe primar el bajo costo de la evaluación sin menoscabo de la calidad del mismo proceso. De esta forma, el presente trabajo pretende dar a conocer un proceso de evaluación de usabilidad (una combinación de métodos) que se ha aplicado en diversos proyectos de investigación realizados al interior de esos grupos.
Este estudio de usabilidad se inscribe en un grupo de trabajos que dan continuidad a una investigación previa en la que se diseñó la plataforma Lingweb [14] y que fue financiado por la Universidad del Valle $^{4}$ en la convocatoria interna de Proyectos de Investigación año 2011. Lingweb es un sistema de administración de cursos de idiomas virtuales, desarrollada colaborativamente entre profesores y estudiantes de la Escuela de Ingeniería de Sistemas y Computación, la Escuela de Ciencias del Lenguaje y el Departamento de Diseño de la Universidad del Valle. La aplicación está basada en una arquitectura multiagente que optimiza la interacción entre usuarios, contenidos y recursos, y en la implementación de agentes pedagógicos inteligentes que cumplen diversas funciones como la ejecución de tareas de escritura así como el seguimiento del desempeño del estudiante en el entorno.

El modelo pedagógico que guió la definición de requerimientos funcionales de Lingweb es de tipo socioconstructivista [15-16] y está fundamentado en un conjunto de hipótesis sobre la adquisición de una segunda lengua, la enseñanza y el aprendizaje de idiomas, a saber principios de orden sociocultural, interaccionista, metacognitivo y colaborativo [1718]. Lingweb promueve formas de aprendizaje donde la construcción de conocimientos de orden lingüístico o cultural, por ejemplo, es el resultado de variables individuales como la estructuración cognitiva de cada aprendiz y sus habilidades para interactuar con las herramientas, los materiales y los contenidos, y de variables sociales como sus habilidades comunicativas y de trabajo con otros. Por otro lado, el enfoque de diseño de cursos en Lingweb se inscribe en una concepción del currículo en acción desde la cual el diseño es entendido como una actividad inacabada e imperfecta que pone el énfasis en la práctica y en los procesos de aprendizaje que ocurren en el aula de clase [19-20], en este caso el aula virtual.

La evaluación de usabilidad de Lingweb, en tanto aplicación para enseñar y aprender idiomas, tiene en cuenta, además de criterios generales de calidad, criterios específicos ligados a las particularidades educativas de la herramienta. $\mathrm{La}$

\footnotetext{
4 Universidad del Valle. Cali, Colombia. http://www.univalle. edu.co/
} 
usabilidad comprende varias capas [21]: técnica, general, académica y específica al contexto que son cruciales en la toma de decisiones pedagógicas durante el diseño de una aplicación educativa o un sistema de e-learning. Así, la evaluación de los aspectos académicos y específicos del contexto de aprendizaje virtual y la evaluación de los contextos de las disciplinas y de las actividades de aprendizaje propias de esas disciplinas deben ser integradas en la evaluación de usabilidad.

Esta evaluación es denominada "usabilidad pedagógica" y viene siendo integrada en las investigaciones sobre usabilidad de aplicaciones educativas [21-23]. Para evaluar la usabilidad pedagógica de Lingweb es necesario verificar en qué medida la herramienta hace posible la realización efectiva y eficiente de las tareas propias del profesor y del aprendiz de idiomas; y qué nivel de satisfacción se observa en uno y otro durante y después de utilizar la aplicación. El aprendizaje de una segunda lengua, por ejemplo, requiere que el ambiente de aprendizaje virtual permita desarrollar de manera eficiente y eficaz las diversas tareas de lectura y escritura, de escucha e interacción oral, según formas variadas de colaboración, permitiendo espacios y proveyendo instrumentos de reflexión metacognitiva, de revisión y retroalimentación entre pares.

\section{METODOLOGÍA DE EVALUACIÓN}

La evaluación de usabilidad de Lingweb se realizó mezclando métodos e instrumentos diversos en varias sesiones de trabajo con los usuarios potenciales de la plataforma: un conjunto de profesores y estudiantes de lenguas (tester-users, en adelante se denominarán TU). Se utilizaron métodos tradicionales de evaluación de usabilidad (evaluación heurística, tasa de cumplimiento de tareas, recorrido cognitivo, tiempo de cumplimiento de tareas, foro de discusión grupal, entrevista grupal y encuesta), los que se mezclaron u originaron nuevos con el ánimo de evaluar no solo los elementos que componen la usabilidad sino la funcionalidad y el aspecto pedagógico ${ }^{5}$. A lo largo de este proceso se generaron insumos que permitieron realizar un análisis riguroso

\footnotetext{
5 Este aspecto se evalúa especialmente en plataformas e-learning conocidas comúnmente como LMS (Learning Management Systems) o CMS (Course management systems).
}

y detallado de las diferentes características de la aplicación informática estudiada.

Generalmente la evaluación de usabilidad se caracteriza por ser un proceso costoso pues requiere una infraestructura equipada con software y hardware especializado en el seguimiento de los TU. Sin embargo, en este estudio se recurrió a un conjunto de técnicas e instrumentos que permiten alcanzar el objetivo de esta experiencia a un menor costo sin perder la calidad del proceso [24]; en este caso, se utilizó, una sala de sistemas equipada con freeware (software de bajo costo).

Para la presente evaluación se partió de la definición propuesta en la Norma ISO 9241 [5], donde la usabilidad se asocia a tres elementos: eficacia, eficiencia y satisfacción. Los elementos de esta triada están en estrecha relación y todos deben estar presentes para asegurar la calidad y la confiabilidad del proceso de evaluación. La selección de los métodos y técnicas se hizo teniendo en cuenta estos tres conceptos y buscando una integración que lograra un análisis más completo de usabilidad. Así pues, esta metodología facilita, no solo la evaluación de la usabilidad per se, sino también evaluaciones de orden funcional y, en este caso, de orden pedagógico debido a las particularidades de la aplicación. De manera que sirve para realizar otros tipos de análisis más robustos en términos de implementación de software e incluso para potenciar procesos pedagógicos en tareas y actividades que implican la interacción con herramientas informáticas.

En el Figura 1 se observa la manera cómo están relacionados los elementos de la usabilidad, los métodos de evaluación y los instrumentos utilizados. En el centro aparecen la eficiencia, la eficacia y la satisfacción; alrededor de esta triada conceptual se presentan los métodos (recuadros) y las técnicas e instrumentos (marcados con flechas); y las relaciones entre usabilidad, funcionalidad y pedagogía están representadas por las líneas continuas o discontinuas dependiendo de la información que se requiera obtener con los instrumentos.

Los métodos de evaluación de usabilidad pueden dividirse en tres grupos [25]: los métodos de inspección, los métodos de indagación y los métodos tipo test. Para responder de manera adecuada a la definición de usabilidad de la ISO se seleccionaron 


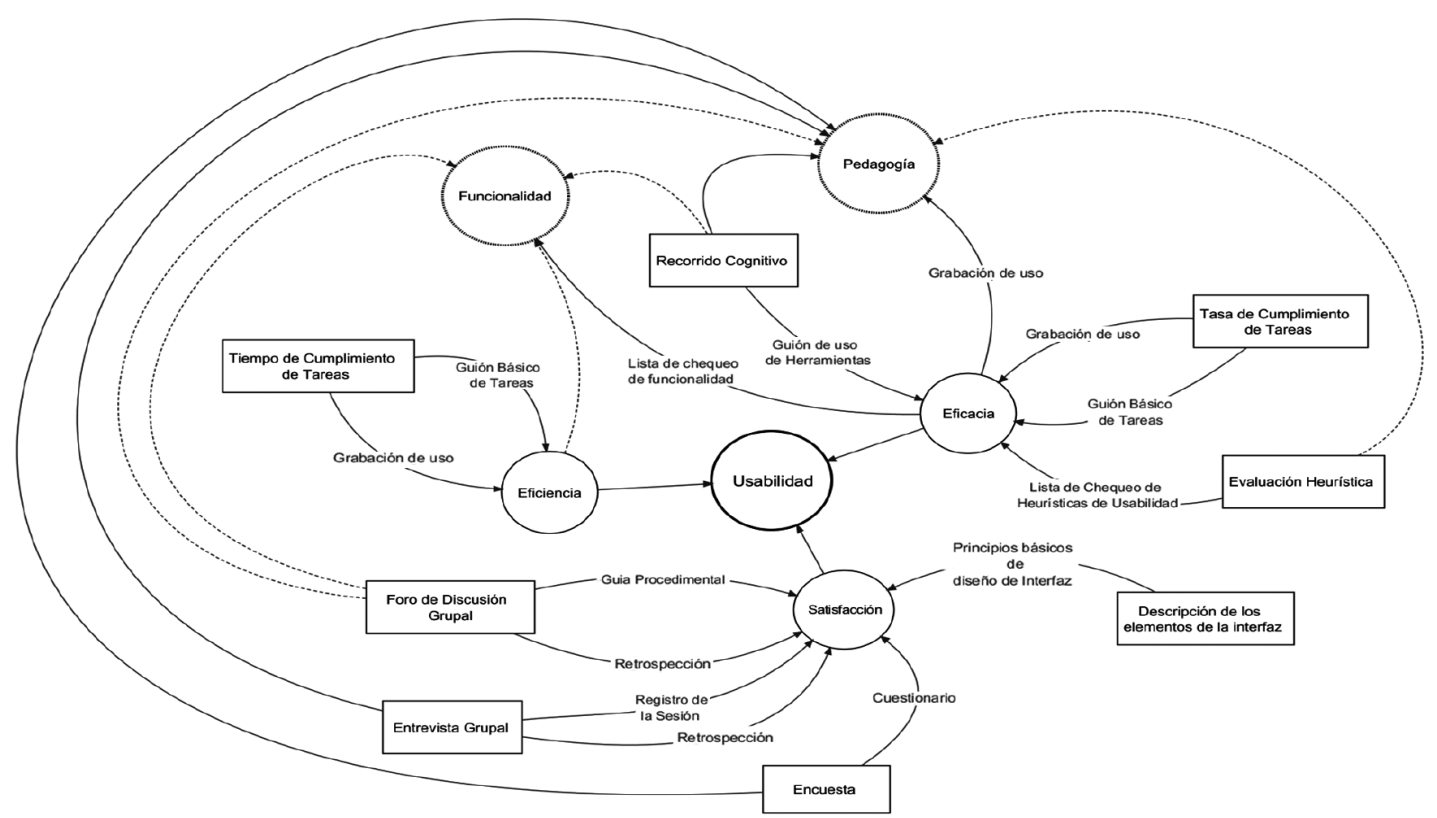

Figura 1. Elementos de usabilidad, métodos y sus relaciones.

algunos métodos de estos tres grupos y se pusieron en relación unos con otros dentro de sus propios límites, lo que permitió cotejar resultados en unas y otras actividades realizadas. En seguida se describe cómo se hizo está combinación para dar cuenta de la triada enunciada ${ }^{6}$.

\section{Evaluación de la Eficiencia}

La eficiencia se define como los recursos empleados en relación con la exactitud y la exhaustividad con las que los usuarios logran las metas (ISO 9241-11) [5]; se basa en la relación del tiempo de cumplimiento para completar las tareas del sistema. En términos de [6], se define como el nivel de desempeño estable cuando el usuario ha superado la curva de aprendizaje.

Para medir la eficiencia, se recurrió al método de análisis del tiempo de cumplimiento de tareas de los usuarios en la plataforma. Se partió de un guion básico de tareas que consiste en un conjunto de actividades que se pueden desarrollar en la plataforma; este instrumento se creó previamente

6 Cuando un mismo método, instrumento o una misma técnica se utilizó para medir más de uno de los elementos de la triada de usabilidad, aquel o aquella se describe en detalle la primera vez que aparece y luego, solo se presenta la justificación de por qué y cómo se utilizó. con la ayuda de expertos en el manejo de la plataforma quienes conocían el tiempo promedio para el desarrollo de cualquier actividad. El guión incluía la descripción general de las acciones que los TU (estudiantes y profesores) debían desarrollar en Lingweb sin sugerir el procedimiento para realizarla, se incluía el tiempo de cada tarea con el propósito de indicar su nivel de dificultad o complejidad.

En el guion básico se plantean tareas como la creación de un curso o de una actividad, la realización de ejercicios, el uso de las herramientas y la actualización de los perfiles de usuario, subir un archivo o cambiar el tema.

El desarrollo de las actividades por parte de cada TU se registró en video a través de la herramienta Screen Recorder®. Esta grabación de uso permitió ir registrando de manera no invasiva las acciones de los usuarios, lo que facilitó que se desempeñaran de manera natural tanto en la plataforma como con las actividades planteadas en el guion. Posteriormente se hizo la observación detallada de cada uno de los TU y se analizaron los tiempos de desarrollo de cada actividad. El cálculo del tiempo no tuvo en cuenta las interrupciones de la actividad por ausencias momentáneas del TU de la sala donde 
se realizó la prueba. Sin embargo, en la prueba se trató de evitar al máximo cualquier interferencia que la afectara, como el uso de dispositivos móviles, lo que garantizó que los TU pudieran realizar las actividades en el tiempo dispuesto. Lo anterior fue expresado en el documento de consentimiento informado que firmaron los participantes.

Por otra parte, se hizo la categorización de los $T U$ en tres tipos: expertos, principiantes y casuales; de acuerdo con su ubicación en la curva de aprendizaje y su dominio de la aplicación [2]. Para ello se aplicó una encuesta de categorización que incluía aspectos relacionados con el uso y la frecuencia de uso de Lingweb y datos sociodemográficos.

Además de permitir analizar los tiempos de realización de tareas, según los tipos de usuarios, la grabación de uso permitió hacer inferencias sobre la tasa de cumplimiento de tareas. El tiempo de cumplimiento de tareas definió en segundos la duración de cada una de las tareas desarrolladas por cada TU. Esta observación se consignó en una matriz cuyas ordenadas son cada una de las tareas planteadas en el guion y las abscisas corresponden a los usuarios. Esta observación permitió definir otro aspecto denominado tasa de cumplimiento con el que se estableció si las tareas fueron o no terminadas a cabalidad en la sesión.

\section{Evaluación de la Eficacia}

La eficacia se define, según la Norma ISO 9241 [5], como el grado de consecución de una meta en la que se mide la efectividad en relación con los objetivos y la exactitud e integridad de los resultados. De esta manera, el elemento para medir la eficacia será el objetivo; una aplicación es eficaz si el usuario puede llevar a cabo la tarea fijada según los criterios establecidos en el objetivo. Para medir la eficacia, se utilizaron tres métodos de análisis: la tasa de cumplimiento de tareas, el recorrido cognitivo [12] y la evaluación heurística $[2,7,11]$.

La tasa de cumplimiento de tareas permitió registrar el proceso de interacción de cada uno de los TU con la aplicación mientras realizaba las actividades propuestas en el guion básico de tareas. La grabación de uso permitió registrar las acciones de los usuarios para luego medir e identificar si los TU pudieron desarrollar la tarea asignada de manera eficaz. Los resultados de estos registros fueron consignados en una matriz que describe la relación entre los usuarios y el porcentaje de éxito en el cumplimiento de tareas.

Por otro lado, el recorrido cognitivo es un método que permite evaluar la facilidad de aprendizaje y el uso de las principales funciones de la aplicación. Es una técnica cualitativa que se basa en el análisis de expertos por medio de la exploración de cada uno de los elementos de la interfaz. En el caso de Lingweb se hizo un recorrido con base en la secuencia natural de uso de las herramientas y del módulo de diseño de cursos, lo que permitió estudiar la granularidad de cada una de las acciones del usuario. Se recurrió a un guion de uso de las herramientas para definir la organización de la exploración de la interfaz; este guion puede ser reemplazado por la ayuda de la aplicación.

Finalmente, la evaluación heurística permitió analizar la aplicación con base en unos principios de usabilidad reconocidos que son denominados heurísticas. Para este propósito se crearon las listas de chequeo de heurísticas de usabilidad con base en las heurísticas planteadas por $[2,6,11]$ y en las heurísticas para aplicaciones e-learning planteadas por [26]. En la Tabla 1 se presenta una breve explicación de cada una de las heurísticas utilizadas en este estudio y en la Figura 2, un ejemplo de una de las listas de chequeo utilizadas. En esta evaluación participaron dos estudiantes de último año de la Licenciatura en Idiomas de la Universidad del Valle, un diseñador industrial experto en usabilidad, una profesora en lenguas experta en pedagogía y multimedia y una ingeniera de sistemas experta en sistemas e-learning.

Después de la generación del reporte de observaciones se establecieron el porcentaje de cumplimiento total para las heurísticas propuestas con el fin de hacer un análisis más esquemático de las tendencias. Este informe de tendencias se alimentó con las observaciones cualitativas generando conclusiones que se pueden evidenciar con mayor claridad. Finalmente, se hizo un procesamiento estadístico para ofrecer una mirada cuantitativa más genérica y, al mismo tiempo, visual de las heurísticas presentadas en las listas de chequeo respecto a las tendencias en el cumplimiento máximo en la aplicación evaluada (escala denominada Excelente). Para ello se analizó 
Tabla 1. Heurísticas de usabilidad utilizadas en la evaluación.

\begin{tabular}{|c|c|}
\hline Heurística & Descripción \\
\hline H0. Generalidades respecto a CMS & $\begin{array}{l}\text { Las plataformas e-learning deben abordar unos elementos } \\
\text { mínimos para ser consideradas como tal. }\end{array}$ \\
\hline H1. Visibilidad del estado del sistema & $\begin{array}{l}\text { El sistema debe mantener siempre a los usuarios informados } \\
\text { del estado del sistema, con una apropiada retroalimentación } \\
\text { y en un tiempo razonable. }\end{array}$ \\
\hline H2. Lenguaje del usuario & $\begin{array}{l}\text { El sistema debe hablar el lenguaje de los usuarios con las } \\
\text { palabras, frases y conceptos familiares, en lugar de que los } \\
\text { términos estén orientados al sistema. }\end{array}$ \\
\hline H3. Control y libertad para el usuario & $\begin{array}{l}\text { Los usuarios eligen a veces funciones del sistema por error } \\
\text { y necesitan a menudo una salida de emergencia claramente } \\
\text { marcada. }\end{array}$ \\
\hline H4. Consistencia y estándares & $\begin{array}{l}\text { Los usuarios no deben tener que preguntarse si las diversas } \\
\text { palabras, situaciones o acciones significan la misma cosa. }\end{array}$ \\
\hline $\begin{array}{l}\text { H5. Ayuda para reconocer, diagnosticar y } \\
\text { solucionar problemas }\end{array}$ & $\begin{array}{l}\text { Los mensajes de error se deben expresar en un lenguaje claro } \\
\text { (sin códigos extraños) indicando exactamente el problema y } \\
\text { siendo constructivos. }\end{array}$ \\
\hline H6. Prevención de errores & $\begin{array}{l}\text { Prevenir la aparición de errores, resulta mejor que generar } \\
\text { buenos mensajes de error. }\end{array}$ \\
\hline H7. Reconocimiento antes que recordación & $\begin{array}{l}\text { El usuario no deberá memorizar la información completa } \\
\text { de la interacción. }\end{array}$ \\
\hline H8. Flexibilidad y eficiencia de uso & $\begin{array}{l}\text { Las instrucciones para el uso del sistema, deben ser visibles } \\
\text { o fácilmente accesibles cada vez que se necesiten. }\end{array}$ \\
\hline H9. Estética de diálogos y diseño minimalista & $\begin{array}{l}\text { No debe existir información que sea irrelevante o que } \\
\text { escasamente se necesite. }\end{array}$ \\
\hline H10. Ayuda general y documentación & $\begin{array}{l}\text { Es necesario disponer de una documentación que sea fácil } \\
\text { de buscar, esté centrada en las tareas del usuario y que no } \\
\text { sea muy extensa. }\end{array}$ \\
\hline H11. Particularidades del usuario & $\begin{array}{l}\text { Un sistema debe tener en cuenta las acciones que puede } \\
\text { ejecutar un usuario para personalizar su apariencia. }\end{array}$ \\
\hline
\end{tabular}

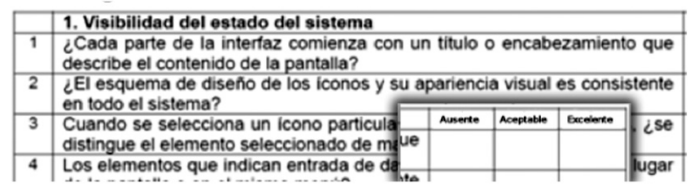

Figura 2. Ejemplo de lista de chequeo utilizada.

cada uno de estos formatos de heurísticas en la sesión grupal y se acordó el cumplimiento a través de porcentajes.

Otro instrumento que apoyó la evaluación de la eficacia en términos de la funcionalidad fueron las listas de chequeo de funcionalidad. Estas se crearon a partir de los requisitos funcionales a evaluar. Están formadas por una serie de ítemes expresados en forma de preguntas que permiten confirmar la aceptación o no del cumplimiento del requisito.

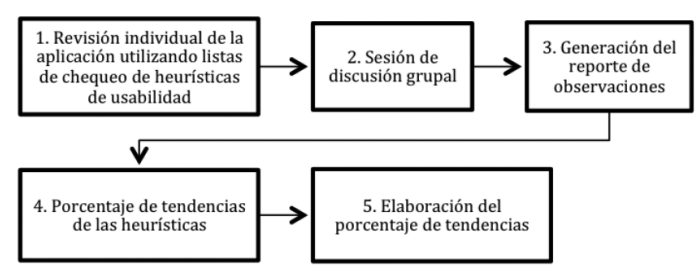

Figura 3. Proceso de evaluación heurística.

Durante el proceso de ejecución de estas pruebas fue necesario hacer diseños instruccionales básicos de las actividades a desarrollar por el estudiante y el profesor; dichos diseños constituyen una especie de escenario de prueba que describe el contexto educativo a evaluar.

El desarrollo de estas pruebas integró a analistas funcionales, testers y usuarios finales quienes 
participaron activamente en el diseño, ejecución y análisis de las pruebas. En la Figura 4 se presenta un ejemplo de una lista de chequeo.

La relación entre la eficacia y la funcionalidad puede observarse en términos del apoyo que brinda la funcionalidad para que un usuario pueda cumplir la tarea propuesta. En este sentido, los errores que puedan existir en la funcionalidades de la aplicación pueden influir significativamente en el cumplimiento y desarrollo de las tareas propuestas. De igual manera en la tasa de cumplimiento de tareas, así como del recorrido cognitivo, se pueden hallar algunos errores funcionales que se deducen implícitamente sin estar realizando una evaluación funcional.

\begin{tabular}{|c|c|c|c|}
\hline $\begin{array}{l}\text { Hardware } \\
\text { requerido }\end{array}$ & \multicolumn{3}{|c|}{$\begin{array}{l}\text { Equipo en donde esté instalada la aplicación - servidor de Pruebas } \\
\text { LINGWEB - }\end{array}$} \\
\hline $\begin{array}{l}\text { Software } \\
\text { requerido }\end{array}$ & \multicolumn{3}{|c|}{ No se requiere ningún software. } \\
\hline Procedimiento & \multicolumn{3}{|c|}{$\begin{array}{l}\text { - Ejecutar en un navegador la aplicación } \\
\text { - Ingresar al sistema como usuario estudiante. } \\
\text { - Entrar a un curso (secuencia 1). } \\
\text { - Entrar a una actividad (secuencia 1). } \\
\text { - Entrar a un ejercicio tipo Apareamiento. } \\
\text { - Evaluar los requerimientos a probar. }\end{array}$} \\
\hline \multicolumn{4}{|c|}{ LISTA DE VERIFICACIÓN } \\
\hline \multicolumn{2}{|c|}{ DETALLE } & \multicolumn{2}{|c|}{$\begin{array}{l}\text { RESULTADO } \\
\end{array}$} \\
\hline \multicolumn{2}{|c|}{$\begin{array}{l}\text { 1. ¿El ejercicio de apareamiento } \\
\text { fue entendible? }\end{array}$} & $\begin{array}{l}\text { S1 } \\
\text { No } \\
\text { PENDIENTE }\end{array}$ & $\begin{array}{l}\text { OBSERVACIONES } \\
\text { Se debería manejar un enunciado corto del } \\
\text { ejercicio, para saber a la segura, que es lo que se } \\
\text { debe hacer. }\end{array}$ \\
\hline \multicolumn{2}{|c|}{$\begin{array}{l}\text { 2. ¿El funcionamiento del } \\
\text { ejercicio fue claro? }\end{array}$} & $\begin{array}{l}\text { SI } \\
\text { No } \\
\text { PENDIENTE }\end{array}$ & $\begin{array}{l}\text { OBSERVACIONES } \\
\text { En el funcionamiento de aparear si es claro, pero } \\
\text { se debería manejar un enunciado corto v el } \\
\text { número de intentos no fue notorio. }\end{array}$ \\
\hline \multicolumn{2}{|c|}{$\begin{array}{l}\text { 3. ¿Entendió en conjunto los } \\
\text { elementos de la interfaz? }\end{array}$} & $\begin{array}{ll}\text { SI } \\
\text { No } \\
\text { PENDIENTE }\end{array}$ & \\
\hline
\end{tabular}

Figura 4.Ejemplo lista de chequeo funcional.

\section{Evaluación de la Satisfacción}

La satisfacción describe la utilidad percibida al interior del sistema de parte del usuario; es el nivel de comodidad al utilizar determinado producto [5]. Este aspecto de usabilidad es mucho más subjetivo y difícil de medir comparado con los dos anteriores; sin embargo, en muchos casos puede ser considerado como el parámetro más importante. La satisfacción se convierte en un factor decisivo para los productos cuyo uso es voluntario [6], por su parte, habla de la satisfacción subjetiva refiriéndose a cuán placentero es usar un sistema específico para un usuario.

En este estudio se utilizaron cuatro métodos para medir la satisfacción a los usuarios al respecto de la aplicación: un foro de discusión grupal con TU-profesores, una entrevista grupal con TU-estudiantes, un análisis de la interfaz de la aplicación y una encuesta.
El foro o grupo de discusión grupal se realizó el 20 de septiembre de 2011 con la participación de ocho $\mathrm{TU}$, cinco profesores y tres estudiantes de último año de la Licenciatura en Lenguas Extranjeras de la Universidad del Valle. El grupo de discusión fue guiado por tres moderadores (los tres profesoresinvestigadores). El propósito fue indagar sobre las características de usabilidad y funcionalidad de Lingweb y recoger las opiniones de los docentes respecto de la interacción con el ambiente virtual. Para iniciar, uno de los moderadores realizó una breve descripción de la actividad y les informó a los TU que no buscaba evaluarlos a ellos sino a la aplicación.

Previamente se había preparado una guía procedimental en la que se establecieron los parámetros de trabajo de la reunión: en la primera parte de la reunión los docentes realizaron una tarea de exploración de Lingweb y una tarea de creación de un curso y de diseño una actividad para ese curso; en la segunda parte se abrió la discusión con una guía de preguntas para evaluar la aplicación. Para la discusión se utilizó como técnica de indagación la retrospección (inmediate recall) de modo que los TU analizaran su interacción con Lingweb en el marco de las tareas que acababan de realizar. Toda la sesión de trabajo y la discusión se registraron en video (registro de la sesión), lo que permitió buscar indicios de conformidad e inconformidad de los TU con la aplicación.

En cuanto a la entrevista grupal a un grupo de TU-estudiantes esta se realizó el 7 de diciembre de 2012. Participaron 15 estudiantes de un curso de francés de la Licenciatura en lenguas quienes habían utilizado la plataforma en varias sesiones durante cerca de dos meses. Con el foro de discusión se buscó indagar, de manera restrospectiva, acerca de la interacción de los estudiantes con Lingweb. Durante la entrevista se hicieron preguntas para establecer los aspectos favorables y desfavorables de la aplicación desde una mirada individual y grupal. El resultado de este proceso fue usado para medir la satisfacción de los usuarios.

La descripción de los elementos de la interfaz es un método empírico y cualitativo basado en una serie de preguntas que buscan analizar la interfaz de una aplicación y su constante relación con los usuarios. Este método da indicios de la satisfacción de los 
usuarios con la aplicación a partir del análisis de las diversas instancias de interacción en las acciones del usuario mientras desarrolla una tarea y del flujo de la información. Este método se desarrolló al interior del grupo CAMALEÓN ${ }^{7}$ con la finalidad de reforzar otros métodos comúnmente usados en las prácticas de evaluación de usabilidad.

Los aspectos que se estudiaron fueron los siguientes: ubicación de los ítemes; establecimiento de niveles de importancia; fluidez de navegación en la información; títulos en ventanas; categorización y organización de información; formularios y aprovechamiento del espacio; íconos; subida de archivos.

En cuanto a la encuesta, esta permitió medir el nivel de satisfacción de los usuarios de la plataforma. El cuestionario fue respondido por los estudiantes de un curso de francés del Departamento de Lenguas Extrajeras de la Escuela de Ciencias del Lenguaje que utilizaron la plataforma en el año 2012.

\section{RESULTADOS}

Los resultados que se presentan en este artículo están basados en la evaluación de la usabilidad en términos de la eficiencia de la aplicación. Desde este punto de vista, la evaluación de la plataforma se basó en el análisis minucioso de los videos, los que presentaban unos tiempos particulares de ejecución de las tareas planteadas en el guion. De esta forma se realizaron los respectivos promedios que permitieron evidenciar la completitud de la tarea. La razón de este último aspecto se dió porque lo que se buscaba era que el usuario respondiera cabalmente a la tarea planteada.

A continuación se presenta una síntesis del análisis realizado a partir de los tiempos de duración de las actividades planteadas a los TU para la evaluación.

\section{Resultados del tiempo de cumplimiento de tareas} Para realizar el análisis relacionado con la eficiencia de la plataforma se utilizó un guion que permitió observar el comportamiento de los usuarios nóveles de Lingweb con base en un conjunto de tareas básicas pero heterogéneas. De esta forma, de parte

7 Grupo de investigación en usabilidad y adaptatividad perteneciente a la Escuela de Ingeniería de Sistemas y Computación de la Universidad del Valle. del experto se sugirió un tiempo para cada conjunto de actividades establecidas en la Tabla 2.

Tabla 2. Relación tiempo sugerido - Tareas.

\begin{tabular}{|c|l|}
\hline $\begin{array}{c}\text { Tiempo sugerido } \\
\text { en minutos }\end{array}$ & \multicolumn{1}{|c|}{ Tareas } \\
\hline 5 & $\mathrm{~T} 1, \mathrm{~T} 2, \mathrm{~T} 3, \mathrm{~T} 4 \mathrm{y} \mathrm{T} 5$ \\
\hline 10 & $\mathrm{~T} 6, \mathrm{~T} 7, \mathrm{~T} 8 \mathrm{y} \mathrm{T} 9$ \\
\hline 10 & $\mathrm{~T} 10 \mathrm{y} \mathrm{T} 11$ \\
\hline 25 & $\mathrm{~T} 12, \mathrm{~T} 13, \mathrm{~T} 14, \mathrm{~T} 15$ y T16 \\
\hline
\end{tabular}

De acuerdo con los resultados de la prueba, el tiempo promedio que se obtuvo para la realización de las tareas se describe en la Tabla 3.

Tabla 3. Relación tiempo promedio - Tareas.

\begin{tabular}{|c|l|}
\hline $\begin{array}{c}\text { Tiempo promedio } \\
\text { en minutos }\end{array}$ & \multicolumn{1}{c|}{ Tareas } \\
\hline 20,34 & $\mathrm{~T} 1, \mathrm{~T} 2, \mathrm{~T} 3, \mathrm{~T} 4$ y T5 \\
\hline 6,35 & $\mathrm{~T} 6, \mathrm{~T} 7, \mathrm{~T} 8$ y T9 \\
\hline 3,07 & $\mathrm{~T} 10 \mathrm{y} \mathrm{T} 11$ \\
\hline 16,43 & $\mathrm{~T} 12, \mathrm{~T} 13, \mathrm{~T} 14, \mathrm{~T} 15$ y T16 \\
\hline
\end{tabular}

Para realizar un análisis detallado de los datos, se han propuesto tres intervalos que permiten agrupar los resultados en tres distintas categorías tal como se muestra en la Tabla 4. En la ecuación $x$ es el valor en minutos sugerido por el experto.

Tabla 4. Intervalos que definen la eficiencia.

\begin{tabular}{|c|c|c|}
\hline$\leq \mathrm{x}-2$ & $(\mathrm{x}-2) \leq \mathrm{x} \leq(\mathrm{x}+2)$ & $\geq \mathrm{x}+2$ \\
\hline Excelente & Aceptable & Deficiente \\
\hline
\end{tabular}

Con base en la inferencia presentada en la Tabla 5 , se puede anotar que para un usuario novel (teniendo en cuenta que debe superar una curva de aprendizaje para adaptarse a la plataforma) le es relativamente fácil interactuar con la plataforma pues de cuatro grupos de tareas que debían realizar tres de ellas las hizo de forma excelente en cuanto al intervalo de tiempo planteado por el experto. Esto indica que, aunque la plataforma Lingweb requiere algunos ajustes que mejoren la eficiencia de las tareas, el uso de las características básicas de su interfaz (ventanas, paneles, ajuste de contenidos, diagramación y jerarquización de elementos de interacción e información) benefician la interacción. 
Tabla 5. Relación tiempo promedio y definición.

\begin{tabular}{|l|c|l|}
\hline Tiempo promedio en minutos & Valor de $\mathbf{X}$ & Definición \\
\hline 20,34 (T1, T2, T3, T4 y T5) & 5 & Deficiente \\
\hline 6,35 (T6, T7, T8 y T9) & 10 & Excelente \\
\hline 3,07 (T10 y T11) & 10 & Excelente \\
\hline 16,43 (T12, T13, T14, T15 y T16) & 25 & Excelente \\
\hline
\end{tabular}

Desde el punto de vista de la usabilidad pedagógica, en general, las tareas planteadas pueden ser desarrolladas de manera eficiente y los objetivos de aprendizaje se logran; el sistema ofrece flexibilidad para la construcción de conocimientos lingüísticos y el desarrollo de habilidades comunicativas; sin embargo, algunos problemas funcionales y de interfaz interfieren a veces en el desempeño de los aprendices en las tareas dentro de la plataforma.

En cuanto a la funcionalidad se observó que, a pesar de que los paneles son una buena solución desde la perspectiva del diseño y agrupación de la información, cuando son muchos suelen amontonarse y generar problemas de visualización, por lo que es necesario realizar un rediseño que involucra aspectos relacionados con el uso de librerías que permitan agrupar y jerarquizar mejor esa información de parte de los usuarios.

\section{CONCLUSIONES}

Los métodos de evaluación que se utilizaron no son puros, es decir, no se aplica un solo método para una sola prueba, sino que se combinan y se complementan entre sí. Lo importante es saber utilizar cada uno de los métodos para los objetivos planteados y que los instrumentos sirvan como puente para realizar otro tipo de análisis.

La metodología propuesta en el proyecto de investigación contribuirá a la revisión de la conceptualización [5] de la usabilidad; sin embargo, el aporte del proyecto va más allá dado que, a partir del uso de las técnicas que permiten evaluar los tres pilares de la usabilidad, también se evalúan los aspectos pedagógicos y funcionales de la plataforma.

Aunque no se necesita de una infraestructura costosa, es importante tener acceso a unos recursos mínimos que permitan realizar las diversas pruebas con los TU, esto es, una sala de cómputo equipada con la aplicación (software) que se va a evaluar.
Además, se requiere de programas y equipos que permitan hacer el seguimiento de las acciones de los TU mientras desarrollan las tareas planteadas en el guion.

Una evaluación de usabilidad debe combinar la interpretación cualitativa con la cuantitativa. $\mathrm{La}$ primera se recomienda para el levantamiento de requerimientos de futuras versiones o ajustes que se hagan dentro del proceso de desarrollo; la segunda, es recomendable cuando se trata de comparar varias versiones o diversas plataformas que tengan objetivos similares.

La forma de evaluar la eficiencia permite generar unos criterios genéricos y a la vez objetivos con la finalidad de medir, en términos concretos, el estado actual de la plataforma; este método resulta ser muy efectivo pero al mismo tiempo es costoso con relación a la cantidad de detalles que se requiere observar en los videos recopilados, por esa razón a futuro se propone el desarrollo de herramientas informáticas que permitan automatizar este análisis.

\section{AGRADECIMIENTOS}

Los autores agradecen a la Vicerrectoría de Investigaciones de la Universidad del Valle y a las Facultades de Ingeniería, Humanidades y Artes Integradas de la misma institución.

\section{REFERENCIAS}

[1] R. Pressman. "Software Engineering: A practitioner's approach". 5a Ed. Mac-Graw Hill. 2001.

[2] B. Shneiderman y C. Plaisant. "Diseño de interfaces de usuario". Pearson Education. Madrid, España. 2006.

[3] ISO 25010. SQuaRE. "Software Engineering - Software product Quality Requirements and Evaluation Quality model”. ISO Press. 2009.

[4] Al-Wabil and H.A. Al-Khalifa. "Framework for Integrating Usability Evaluations Methods: The Mawhiba Web Portal Case Study". 2009.

[5] ISO 9241-11. "International Standard Organization ISO 9241-11. Ergonomic requirements for office work with visual display terminals. Part 11: Guidance on usability". 1998. 
[6] J. Nielsen. "Usability Engineering". $1^{\text {a }}$ Ed. United States of America. Academic Press. 1993.

[7] J. Reyes and A. Libreros. "Integral evaluation method of usability for e-learning systems". Revista Educación en Ingeniería ACOFI, 12, 69-79. 2011.

[8] A. Følstad, E. Lai-Chong and K. Hornbæk. "Analysis in practical usability evaluation: a survey study". CHI 2012. Austin, Texas, USA. ACM. May 5-10, 2012.

[9] T. Babaian, W. Lucas and M. Oja. "Evaluating the Collaborative Critique Method". CHI 2012. Austin, Texas, USA. ACM. May 5-10, 2012.

[10] J. Derboven, D. DeRoeck and M. Verstraete. "Semiotic analysis of multi-touch interface design: The MuTable case study". International Journal of Human-Computer Studies. Vol. 70 Issue 10, pp. 714-728. October, 2012.

[11] R. Molich. "Improving a human-computer dialogue". Communications of the ACM 33, pp. 338-348. 1990.

[12] L. Rivero and T. Conte. "Using an Empirical Study to Evaluate the Feasibility of a New Usability Inspection Technique for Paper Based Prototypes of Web Applications". Conferences Publishing Services. IEEE. 2012.

[13] B. Florian, O. Solarte and J. Reyes. "Propuesta para incorporar evaluación y pruebas de usabilidad dentro de un proceso de desarrollo de software". Revista EIA. Vol. 13, pp. 123-141. 2010.

[14] M. Berdugo and N. Pedraza. "Ambiente virtual basado en agentes software para el aprendizaje de lenguas". Informe final de investigación (inédito). Cali: Universidad del Valle. 2010.

[15] J. Delval. "Tesis sobre el constructivismo". En M.J. Rodrigo y J. Arnay (Eds.). La construcción del conocimiento escolar, pp. 15-33. Paidós. Barcelona, España. 1997.

[16] M. Carretero y M. Limón. "Problemas actuales del constructivismo. De la teoría a la práctica". En M-J. Rodrigo y J. Arnay (Eds.). La construcción del conocimiento escolar (pp. 137-153). Paidós. Barcelona, España. 1997.
[17] M. Berdugo y N. Pedraza. "Construcción de un ambiente web para la enseñanza y el aprendizaje de idiomas". Cali: Unidad de Artes Gráficas Humanidades, Universidad del Valle. 2005.

[18] M. Berdugo y N. Pedraza. "Evaluación de Lingweb, ambiente virtual para el aprendizaje de lenguas". Lenguaje. Vol. $36 \mathrm{~N}^{\circ} 2$, pp. 475 511. 2008.

[19] K. Graves. "The language curriculum: A social context perspective". Language Teaching. Vol. $41 \mathrm{~N}^{\circ}$ 2, pp. 147-181.2008.

[20] K. Graves. "Designing Language Courses. A guide for teachers". Heinle\&Heinle. Boston, USA. 2000.

[21] L. Shield and A. Kukulska-Hulme. "Are language learning websites special? Toward a Research Agenda for Discipline-Specific Usability". Journal of Educational Multimedia and Hypermedia. Vol. 15 Issue 3, pp. 349369. 2006.

[22] A. Kukulska-Hulme and L. Shield. "Usability and Pedagogical Design: are Language Learning Websites Special?'. Paper presented at ED-Media 2004. Lougano, Suisse. Centre pour la Communication Scientifique Directe (CCSD), Centro Nacional de Investigación Científica (CNRS). Francia. URL: http:// hal.archives-ouvertes.fr. 2004.

[23] M. Maris, A. De Giuste y P. Pesado. "Métodos de evaluación de usabilidad: una propuesta de aplicación en Objetos de Aprendizaje”. XIV Workshop de Investigadores en Ciencias de la Computación (WICC). 2012.

[24] E. Van Veenendaal. "Low cost usability Testing. Software quality and software testing in internet times", pp. 153-164. SpringerVerlag, Inc. New York, NY, USA. 2002.

[25] M.S. Mahrin. "Selecting Usability Evaluation Methods for Software Process Descriptions". 16th Asia-Pacific Software Engineering Conference. IEEE, pp. 523-529. 2009.

[26] L. Dringus \& M. Cohen, M. "An Adaptable Usability Heuristic Checklist for Online Courses". 35th ASEE/IEEE Frontiers in Education. IEEE. Indianapolis, IN. October 19-22, 2005. 